\title{
HOMOLOGICAL ASPECTS OF SEMIDUALIZING MODULES
}

\author{
RYO TAKAHASHI and DIANA WHITE* \\ (Dedicated to the memory of Anders J. Frankild)
}

\begin{abstract}
We investigate the notion of the $C$-projective dimension of a module, where $C$ is a semidualizing module. When $C=R$, this recovers the standard projective dimension. We show that three natural definitions of finite $C$-projective dimension agree, and investigate the relationship between relative cohomology modules and absolute cohomology modules in this setting. Finally, we prove several results that demonstrate the deep connections between modules of finite projective dimension and modules of finite $C$-projective dimension. In parallel, we develop the dual theory for injective dimension and $C$-injective dimension.
\end{abstract}

\section{Introduction}

Grothendieck [6] introduced dualizing modules as tools for investigating cohomology theories in algebraic geometry. In this paper, we investigate semidualizing modules and associated relative cohomology functors. Foxby [4], Vasconcelos [12] and Golod [5] independently initiated the study of semidualizing modules. Over a noetherian ring $R$, a finitely generated $R$-module $C$ is semidualizing if the natural homothety map $R \rightarrow \operatorname{Hom}_{R}(C, C)$ is an isomorphism and $\operatorname{Ext}_{R}^{\geqslant 1}(C, C)=0$; see 1.2 for a more general definition. Examples include a dualizing module, when one exists, and all finitely generated rank 1 projective modules.

Throughout this introduction, let $R$ be a commutative ring and $C$ a semidualizing $R$-module. The class of $C$-projectives, denoted $\mathscr{P}_{C}$, consists of those $R$-modules of the form $C \otimes_{R} P$ for some projective $R$-module $P$. These form the building blocks of the so-called $G_{C}$-projectives, which are studied in depth in [13]. Every $R$-module $M$ admits an augmented proper $\mathscr{P}_{C}$-projective resolution. That is, there exists a complex

$$
X^{+}=\cdots \rightarrow C \otimes_{R} P_{n} \rightarrow \cdots \rightarrow C \otimes_{R} P_{0} \rightarrow M \rightarrow 0
$$

\footnotetext{
* This research was conducted in part while R. T. visited the University of Nebraska in August 2006, partly supported by NSF grant DMS 0201904.

Received 7 November 2007.
} 
such that the complex

$$
\operatorname{Hom}_{R}\left(C, X^{+}\right)=\cdots \rightarrow P_{n} \rightarrow \cdots \rightarrow P_{0} \rightarrow \operatorname{Hom}_{R}(C, M) \rightarrow 0
$$

is exact. Despite the fact that these augmented proper $\mathscr{P}_{C}$-resolutions may not be exact, they still have particularly good lifting properties. In particular, they give rise to well-defined cohomology modules $\operatorname{Ext}_{\mathscr{P}_{C}}^{i}(M, N)$ for all $R$-modules $M$ and $N$; see 1.5. In the case $C=R$, these notions recover the projectives, projective resolutions and the "absolute" cohomology $\operatorname{Ext}_{R}^{i}(M, N)$, respectively.

Because augmented proper $\mathscr{P}_{C}$-projective resolutions need not be exact, it is not immediately clear how best to define the $\mathscr{P}_{C}$-projective dimension of a module. For instance, should one consider arbitrary proper $\mathscr{P}_{C}$-projective resolutions or only exact ones? Or should it be defined in terms of the vanishing of the functors $\operatorname{Ext}_{\mathscr{P}_{C}}^{n}(M,-)$ ? The next result, proved in Corollary 2.10, shows that each of these approaches gives rise to the same invariant.

Theorem A. Let $M$ be an $R$ module. The following quantities are equal.

$$
\begin{aligned}
& \text { (1) } \inf \left\{\begin{array}{l|l}
\sup \left\{n \mid X_{n} \neq 0\right\} & \begin{array}{l}
X^{+} \text {is an augmented proper } \\
\mathscr{P}_{C} \text {-projective resolution of } M
\end{array}
\end{array}\right\} \\
& \text { (2) } \inf \left\{\begin{array}{l|l}
\sup \left\{n \mid X_{n} \neq 0\right\} & \begin{array}{l}
X^{+} \text {is an exact augmented proper } \\
\mathscr{P}_{C} \text {-projective resolution of } M
\end{array}
\end{array}\right\} \\
& \text { (3) } \sup \left\{n \mid \operatorname{Ext}_{\mathscr{P}_{C}}^{n}(M,-) \neq 0\right\}
\end{aligned}
$$

The proof of this result uses so-called Auslander and Bass class techniques; see 1.8 .

Our investigation demonstrates a strong connection between the modules of finite $\mathscr{P}_{C}$-projective dimension and modules of finite projective dimension, which is the focus of Section 2. For example, the following is part of Theorem 2.11.

Theorem B. If $M$ is an $R$-module, then $\mathscr{P}_{C}-\operatorname{pd}_{R}(M)=\operatorname{pd}_{R}\left(\operatorname{Hom}_{R}(C\right.$, $M)$ ).

If $R$ is Cohen-Macaulay and local with dualizing module $D$, then a result of Sharp shows that the modules of finite $\mathscr{P}_{D}$-projective dimension are precisely the modules of finite injective dimension. Thus, this theorem recovers part of the Foxby equivalence from [1], namely that $M$ has finite injective dimension if and only if $\operatorname{Hom}_{R}(D, M)$ has finite projective dimension. 
Section 4 explores the connection between the cohomology functors Ext $\mathscr{P}_{C}$ and $\operatorname{Ext}_{R}$. These connections are used in [9] to distinguish between several different relative cohomology theories. For example, the following is part of Theorem 4.1 and Corollary 4.2. See 1.8 for the definition of the Bass class.

TheOREM C. Let $M$ and $N$ be R-modules. There is an isomorphism $\operatorname{Ext}_{\mathscr{P}_{C}}^{i}(M, N) \cong \operatorname{Ext}_{R}^{i}\left(\operatorname{Hom}_{R}(C, M), \operatorname{Hom}_{R}(C, N)\right)$ for all $i$. If $M$ and $N$ are in the Bass class with respect to $C$, then $\operatorname{Ext}_{\mathscr{P}_{C}}^{i}(M, N) \cong \operatorname{Ext}_{R}^{i}(M, N)$ for all $i$.

This result and several others from this work have already proved valuable for other investigations. For instance, they are used by the second author and her collaborators in [11] to analyze the structure of certain categories naturally associated to semidualizing modules, and in [9], [10] to study balance properties of relative cohomology theories and to distinguish between them. We expect this line of inquiry to continue to shed new light on the relationship between classical and relative homological algebra.

Finally, in Section 5 of the paper we use results from the previous sections to demonstrate the depth of the connection between modules of finite $\mathscr{P}_{C^{-}}$ projective dimension and modules of finite projective dimension.

\section{Preliminaries}

Throughout this paper, let $R$ be a commutative ring.

1.1. An $R$-complex is a sequence of $R$-module homomorphisms

$$
X=\cdots \stackrel{\partial_{n+1}^{X}}{\longrightarrow} X_{n} \stackrel{\partial_{n}^{X}}{\longrightarrow} X_{n-1} \stackrel{\partial_{n-1}^{X}}{\longrightarrow} \cdots
$$

such that $\partial_{n-1}^{X} \partial_{n}^{X}=0$ for each integer $n$; the $n$th homology module of $X$ is $\mathrm{H}_{n}(X)=\operatorname{Ker}\left(\partial_{n}^{X}\right) / \operatorname{Im}\left(\partial_{n+1}^{X}\right)$. A morphism of complexes $\alpha: X \rightarrow Y$ induces homomorphisms $\mathrm{H}_{n}(\alpha): \mathrm{H}_{n}(X) \rightarrow \mathrm{H}_{n}(Y)$, and $\alpha$ is a quasiisomorphism when each $\mathrm{H}_{n}(\alpha)$ is bijective. The complex $X$ is bounded if $X_{n}=0$ for $|n| \gg 0$. It is degreewise finite if each $X_{i}$ is finitely generated.

\subsection{An $R$-module $C$ is semidualizing if}

(a) $C$ admits a degreewise finite projective resolution,

(b) The natural homothety map $R \rightarrow \operatorname{Hom}_{R}(C, C)$ is an isomorphism, and

(c) $\operatorname{Ext}_{R}^{\geqslant 1}(C, C)=0$.

A free $R$-module of rank 1 is semidualizing, and indeed this is the only semidualizing module over a Gorenstein local ring. If $R$ is noetherian, local and admits a dualizing module $D$, then $D$ is semidualizing. 
The next two classes of modules have been studied in numerous papers, see e.g. [2] and [7].

1.3. The classes of $C$-projective and $C$-injective modules are defined as

$$
\begin{aligned}
& \mathscr{P}_{C}=\left\{C \otimes_{R} P \mid P \text { is projective }\right\}, \\
& \mathscr{I}_{C}=\left\{\operatorname{Hom}_{R}(C, I) \mid I \text { is injective }\right\} .
\end{aligned}
$$

When $C=R$, we omit the subscript and recover the classes of projective and injective $R$-modules.

The next four paragraphs provide the necessary background on relative homological algebra. The reader is encouraged to consult [3] for details.

1.4. The class $\mathscr{P}_{C}$ is precovering by [7, (5.10)]. That is, given an $R$-module $M$, there exists a projective module $P$ and a homomorphism $\phi: C \otimes_{R} P \rightarrow M$ such that, for every projective $Q$, the induced map

$$
\operatorname{Hom}_{R}\left(C \otimes_{R} Q, C \otimes_{R} P\right) \stackrel{\operatorname{Hom}_{R}\left(C \otimes_{R} Q, \phi\right)}{\longrightarrow} \operatorname{Hom}_{R}\left(C \otimes_{R} Q, M\right)
$$

is surjective. Dually, the class $\mathscr{I}_{C}$ is preenveloping.

1.5. Since the class $\mathscr{P}_{C}$ is precovering, for any $R$-module $M$ one can iteratively take precovers to construct an augmented proper $\mathscr{P}_{C}$-projective resolution of $M$, that is, a complex

$$
X^{+}=\cdots \stackrel{\partial_{2}^{X}}{\longrightarrow} C \otimes_{R} P_{1} \stackrel{\partial_{1}^{X}}{\longrightarrow} C \otimes_{R} P_{0} \stackrel{\partial_{0}^{X}}{\longrightarrow} M \rightarrow 0
$$

such that $\operatorname{Hom}_{R}\left(C \otimes_{R} Q, X^{+}\right)$is exact for all projective $R$-modules $Q$. The truncated complex

$$
X=\cdots \stackrel{\partial_{2}^{X^{+}}}{\longrightarrow} C \otimes_{R} P_{1} \stackrel{\partial_{1}^{X^{+}}}{\longrightarrow} C \otimes_{R} P_{0} \rightarrow 0
$$

is a proper $\mathscr{P}_{C}$-projective resolution of $M$. For $n \geqslant 0$, set

$$
\Omega_{n}^{X^{+}}= \begin{cases}M & \text { if } n=0 \\ \operatorname{Ker}\left(\partial_{n-1}^{X^{+}}\right) & \text {if } n \geqslant 1\end{cases}
$$

Note that $X^{+}$need not be exact unless $C=R$.

Dually, let $N$ be an $R$-module with augmented proper $\mathscr{I}_{C}$-injective resolution

$$
Y^{+}=0 \rightarrow N \rightarrow \operatorname{Hom}_{R}\left(C, I^{0}\right) \stackrel{\partial_{Y}^{0}}{\longrightarrow} \operatorname{Hom}_{R}\left(C, I^{1}\right) \stackrel{\partial_{Y}^{1}}{\longrightarrow} \cdots
$$


For an integer $n \geqslant 0$, set

$$
\Omega_{Y^{+}}^{n}= \begin{cases}N & \text { if } n=0 \\ \operatorname{Im}\left(\partial_{Y^{+}}^{n-1}\right) & \text { if } n \geqslant 1\end{cases}
$$

Proper $\mathscr{P}_{C}$-projective resolutions are unique up to homotopy equivalence; see e.g. $[8,(1.8)]$. Accordingly, the $n$th relative cohomology modules

$$
\operatorname{Ext}_{\mathscr{P}_{C}}^{n}(M, N)=\mathrm{H}^{n} \operatorname{Hom}_{R}(X, N)
$$

where $X$ is a proper $\mathscr{P}_{C}$-projective resolution of $M$ are well-defined for each integer $n$. The cohomology modules $\operatorname{Ext}_{\mathscr{I}_{C}}^{n}(M, N)$ are defined dually.

1.6. The $\mathscr{P}_{C}$-projective dimension of $M$ is

$$
\mathscr{P}_{C^{-}} \operatorname{pd}(M)=\inf \left\{\begin{array}{l|l}
\sup \left\{n \mid X_{n} \neq 0\right\} & \begin{array}{l}
X \text { is a proper } \mathscr{P}_{C} \text {-projective } \\
\text { resolution of } M
\end{array}
\end{array}\right\}
$$

The modules of $\mathscr{P}_{C}$-projective dimension zero are the non-zero modules in $\mathscr{P}_{C}$. The $\mathscr{I}_{C}$-injective dimension, denoted $\mathscr{I}_{C}-\mathrm{id}(-)$ is defined dually.

1.7 (Dimension Shifting). Let $N$ be an $R$-module. For any augmented proper $\mathscr{P}_{C}$-projective resolution $X^{+}$(as above) of $M$, there are isomorphisms

$$
\begin{aligned}
\operatorname{Ext}_{\mathscr{P}_{C}}^{i}(M, N) \cong \operatorname{Ext}_{\mathscr{P}_{C}}^{i-1}\left(\Omega_{1}^{X^{+}}, N\right) & \\
& \cong \operatorname{Ext}_{\mathscr{P}_{C}}^{i-2}\left(\Omega_{2}^{X^{+}}, N\right) \cong \cdots \cong \operatorname{Ext}_{\mathscr{P}_{C}}^{i-n}\left(\Omega_{n}^{X^{+}}, N\right)
\end{aligned}
$$

for integers $1 \leqslant n<i$.

1.8. The Bass class with respect to $C$, denoted $\mathscr{B}_{C}$ or $\mathscr{B}_{C}(R)$, consists of all $R$-modules $M$ satisfying

(a) $\operatorname{Ext}_{R}^{\geqslant 1}(C, M)=0=\operatorname{Tor}_{\geqslant 1}^{R}\left(C, \operatorname{Hom}_{R}(C, M)\right)=0$, and

(b) The natural evaluation map $v_{C C M}: C \otimes_{R} \operatorname{Hom}_{R}(C, M) \rightarrow M$ is an isomorphism. We will write $v_{M}=v_{C C M}$ if there is no confusion.

Dually, the Auslander class with respect to $C$, denoted $\mathscr{A}_{C}$ or $\mathscr{A}_{C}(R)$, consists of all $R$-modules $M$ satisfying

(c) $\operatorname{Tor}_{\geqslant 1}^{R}(C, M)=0=\operatorname{Ext}_{R}^{\geqslant 1}\left(C, C \otimes_{R} M\right)$, and

(d) The natural map $\mu_{C C M}: M \rightarrow \operatorname{Hom}_{R}\left(C, C \otimes_{R} M\right)$ is an isomorphism. We will write $\mu_{M}=\mu_{C C M}$ if there is no confusion.

We now state some basic results about the classes $\mathscr{A}_{C}$ and $\mathscr{B}_{C}$. These facts are well-known when $R$ is noetherian. In this generality, the first follows 
from [7, (6.5)], the second follows from the first, and the third assertion is routine to check.

1.9. The following hold.

(a) If any two $R$-modules in a short exact sequence are in $\mathscr{A}_{C}$, respectively $\mathscr{B}_{C}$, then so is the third.

(b) The class $\mathscr{A}_{C}$ contains all modules of finite flat dimension. The class $\mathscr{B}_{C}$ contains all modules of finite injective dimension.

(c) If $M$ is in $\mathscr{A}_{C}$, then $C \otimes_{R} M$ is in $\mathscr{B}_{C}$. If $M$ is in $\mathscr{B}_{C}$, then $\operatorname{Hom}_{R}(C, M)$ is in $\mathscr{A}_{C}$.

\section{Relative dimensions and Auslander and Bass classes}

This section has two interwoven themes. First, we explore the interplay between $\mathscr{P}_{C}$-projective dimension and projective dimension. Second, we investigate the exactness of augmented proper $\mathscr{P}_{C}$-projective resolutions for modules of finite $\mathscr{P}_{C}$-projective dimension.

We begin with the following lemma, which follows from the definitions of semidualizing modules and augmented proper resolutions, using 1.9(b).

Lemma 2.1. Let $C$ be a semidualizing $R$-module and $M$ an $R$-module.

(a) If $X^{+}$is an augmented proper $\mathscr{P}_{C}$-projective resolution of $M$, then $\operatorname{Hom}_{R}\left(C, X^{+}\right)$is an augmented projective resolution of $\operatorname{Hom}_{R}(C, M)$.

(b) If $Y^{+}$is an augmented proper $\mathscr{I}_{C}$-injective resolution of $M$, then $C \otimes_{R} Y^{+}$ is an augmented injective resolution of $C \otimes_{R} M$.

We now investigate exactness of augmented proper $\mathscr{P}_{C}$-projective resolutions.

Proposition 2.2. Let $C$ be a semidualizing $R$-module, $M$ an $R$-module and $n$ a nonnegative integer.

(a) The following are equivalent.

(i) There exists an augmented proper $\mathscr{P}_{C}$-projective resolution of $M$ which is exact in degree less than $n$;

(ii) Every augmented proper $\mathscr{P}_{C}$-projective resolution of $M$ is exact in degree less than $n$;

(iii) The natural homomorphism $v_{M}: C \otimes_{R} \operatorname{Hom}_{R}(C, M) \rightarrow M$ is an isomorphism and $\operatorname{Tor}_{i}^{R}\left(C, \operatorname{Hom}_{R}(C, M)\right)=0$ for $0<i<n$.

(b) The following are equivalent.

(i) There exists an augmented proper $\mathscr{I}_{C}$-injective resolution of $M$ which is exact in degree less than $n$; 
(ii) All augmented proper $\mathscr{I}_{C}$-injective resolutions of $M$ are exact in degree less than $n$;

(iii) The natural homomorphism $\mu_{M}: C \rightarrow \operatorname{Hom}_{R}\left(C, C \otimes_{R} M\right)$ is an isomorphism and $\operatorname{Ext}_{R}^{i}\left(C, C \otimes_{R} M\right)=0$ for $0<i<n$.

Proof. We prove only part(a). Let $X^{+}$be an augmented proper $\mathscr{P}_{C}$-projective resolution of $M$. By Lemma 2.1, the complex $\operatorname{Hom}_{R}(C, X)$ is a projective resolution of $\operatorname{Hom}_{R}(C, M)$. Hence, there is an isomorphism $\operatorname{Tor}_{i}^{R}(C$, $\left.\operatorname{Hom}_{R}(C, M)\right) \cong H_{i}\left(C \otimes_{R} \operatorname{Hom}_{R}(C, X)\right)$ for all $i \geqslant 0$. Since each $X_{i}$ is in $\mathscr{B}_{C}$, the natural chain map $C \otimes_{R} \operatorname{Hom}_{R}(C, X) \rightarrow X$ is an isomorphism. The result follows

From the above proposition, we obtain the following criterion for a given module to possess exact augmented proper resolutions.

Corollary 2.3. Let $C$ be a semidualizing $R$-module and $M$ an $R$-module.

(a) The following are equivalent.

(i) $M$ admits an exact augmented proper $\mathscr{P}_{C}$-projective resolution;

(ii) All augmented proper $\mathscr{P}_{C}$-projective resolutions of $M$ are exact;

(iii) The natural homomorphism $v_{M}: C \otimes_{R} \operatorname{Hom}_{R}(C, M) \rightarrow M$ is an isomorphism and $\operatorname{Tor}_{\geqslant 1}^{R}\left(C, \operatorname{Hom}_{R}(C, M)\right)=0$.

(b) The following are equivalent.

(i) $M$ admits an exact augmented proper $\mathscr{I}_{C}$-injective resolution;

(ii) All augmented proper $\mathscr{I}_{C}$-injective resolutions of $M$ are exact;

(iii) The natural homomorphism $\mu_{M}: M \rightarrow \operatorname{Hom}_{R}\left(C, C \otimes_{R} M\right)$ is an isomorphism and $\mathrm{Ext}_{R}^{\geqslant 1}\left(C, C \otimes_{R} M\right)=0$.

From the definitions of the Auslander and Bass classes, we have the following.

Corollary 2.4. Let $C$ be a semidualizing $R$-module and $M$ an $R$-module.

(a) Assume $M$ is in $\mathscr{B}_{C}$. Then every augmented proper $\mathscr{P}_{C}$-projective resolution of $M$ is exact. In particular, every $\mathscr{P}_{C}$-precover of $M$ is surjective.

(b) Assume $M$ is in $\mathscr{A}_{C}$. Then every augmented proper $\mathscr{I}_{C}$-resolution of $M$ is exact. In particular, every $\mathscr{I}_{C}$-preenvelope of $M$ is injective.

The next few technical results build toward the following fact, which follows from Corollaries 2.3 and 2.10: if $M$ has finite $\mathscr{P}_{C}$-projective dimension, then every augmented proper $\mathscr{P}_{C}$-resolution of $M$ is exact.

Lemma 2.5. Let $C$ be a semidualizing module and $M$ an $R$-module.

(a) The composition $\operatorname{Hom}_{R}\left(C, v_{M}\right) \circ \mu_{\mathrm{Hom}_{R}(C, M)}$ is the identity map on $\operatorname{Hom}_{R}(C, M)$. Hence, $\operatorname{Hom}_{R}\left(C, v_{M}\right)$ is a split epimorphism and $\mu_{\mathrm{Hom}_{R}(C, M)}$ is a split monomorphism. 
(b) Assume that $v_{M}$ is injective. The composition $\mu_{\mathrm{Hom}_{R}(C, M)} \circ \operatorname{Hom}_{R}\left(C, v_{M}\right)$ is the identity map on $\operatorname{Hom}_{R}\left(C, C \otimes_{R} \operatorname{Hom}_{R}(C, M)\right)$. Hence, $\operatorname{Hom}_{R}(C$, $\left.v_{M}\right)$ is an isomorphism and $\mu_{\mathrm{Hom}_{R}(C, M)}$ is the inverse isomorphism.

(c) The composition $v_{C \otimes_{R} M} \circ\left(C \otimes_{R} \mu_{M}\right)$ is the identity map on $C \otimes_{R}$ M. Hence, $C \otimes_{R} \mu_{M}$ is a split monomorphism and $\nu_{C \otimes_{R} M}$ is a split epimorphism.

(d) Assume that $\mu_{M}$ is surjective. The composition $\left(C \otimes_{R} \mu_{M}\right) \circ v_{C \otimes_{R} M}$ is the identity map on $C \otimes_{R} \operatorname{Hom}_{R}\left(C, C \otimes_{R} M\right)$. Hence, $C \otimes_{R} \mu_{M}$ is an isomorphism and $\nu_{C \otimes_{R} M}$ is the inverse isomorphism.

Proof. Part (a) is straightforward to check. For part (b), set $\rho=$ $\mu_{\mathrm{Hom}_{R}(C, M)} \circ \operatorname{Hom}_{R}\left(C, v_{M}\right)$. Note that if $\xi \in \operatorname{Hom}_{R}\left(C, C \otimes_{R} \operatorname{Hom}_{R}(C, M)\right)$, then $\rho(\xi)$ sends $z \in C$ to $z \otimes\left(v_{M} \circ \xi\right)$. Thus, $v_{M}\left(\xi(z)-z \otimes\left(v_{M} \cdot \xi\right)\right)=$ $v_{M}(\xi(z))-v_{M}(\xi(z))=0$. Since $v_{M}$ is injective, there is an equality $\xi(z)=$ $z \otimes\left(v_{M} \cdot \xi\right)$, thereby showing that $\rho$ is the identity. Parts (c) and (d) are proved similarly.

Lemma 2.6. Let $C$ be a semidualizing $R$-module and $M$ an $R$-module.

(a) Assume $\operatorname{Ext}_{R}^{1}\left(C, C \otimes_{R} \operatorname{Hom}_{R}(C, M)\right)=0$. If $v_{M}$ is injective, then it is an isomorphism.

(b) Assume that $\operatorname{Tor}_{1}^{R}\left(C, \operatorname{Hom}_{R}\left(C, C \otimes_{R} M\right)\right)=0$. If $\mu_{M}$ is surjective, then it is an isomorphism.

Proof. We prove only part (a), as part (b) is dual. Set $L=\operatorname{Coker} v_{M}$. Applying $\operatorname{Hom}_{R}(C,-)$ to the exact sequence

$$
0 \rightarrow C \otimes_{R} \operatorname{Hom}_{R}(C, M) \stackrel{\nu_{M}}{\longrightarrow} M \rightarrow L \rightarrow 0
$$

induces an exact sequence

$$
\begin{aligned}
0 \rightarrow \operatorname{Hom}_{R}\left(C, C \otimes \operatorname{Hom}_{R}(C, M)\right) \\
\stackrel{\operatorname{Hom}\left(C, v_{M}\right)}{\longrightarrow} \\
\operatorname{Hom}_{R}(C, M) \rightarrow \operatorname{Hom}_{R}(C, L) \rightarrow 0
\end{aligned}
$$

where right-exactness follows from the equality $\operatorname{Ext}_{R}^{1}\left(C, C \otimes_{R} \operatorname{Hom}_{R}(C\right.$, $M))=0$. By Lemma 2.5, $\operatorname{Hom}_{R}\left(C, v_{M}\right)$ is an isomorphism. Hence, the above exact sequence implies that $\operatorname{Hom}_{R}(C, L)=0$, and it follows from [7, (3.6)] that $L=0$.

Corollary 2.7. Let $C$ be a semidualizing $R$-module and $M$ an $R$-module.

(a) Suppose $\operatorname{Hom}_{R}(C, M)$ is in $\mathscr{A}_{C}$. If $v_{M}$ is injective, then it is an isomorphism.

(b) Suppose $C \otimes_{R} M$ is in $\mathscr{B}_{C}$. If $\mu_{M}$ is surjective, then it is an isomorphism. 
We now prove our first theorem, which leads to some of the main results of this section.

Theorem 2.8. Let $C$ be a semidualizing $R$-module and $M$ an $R$-module. Then the following hold.

(a) $M \in \mathscr{B}_{C}$ if and only if $\operatorname{Hom}_{R}(C, M) \in \mathscr{A}_{C}$.

(b) $M \in \mathscr{A}_{C}$ if and only if $C \otimes_{R} M \in \mathscr{B}_{C}$.

Proof. We prove only part (a), as part (b) is proved similarly. According to 1.9(c), it is enough to assume $\operatorname{Hom}_{R}(C, M) \in \mathscr{A}_{C}$ and show $M \in$ $\mathscr{B}_{C}$. The definition of $\mathscr{A}_{C}$ implies that $\operatorname{Tor}_{\geqslant 1}^{R}\left(C, \operatorname{Hom}_{R}(C, M)\right)=0$ and $\operatorname{Ext}_{R}^{\geqslant 1}\left(C, C \otimes_{R} \operatorname{Hom}_{R}(C, M)\right)=0$. We will show that the evaluation map $v_{M}$ is an isomorphism. Using the above vanishings, it will then follow that $\operatorname{Ext}_{R}^{\geqslant 1}(C, M)=0$ and $M$ is in $\mathscr{B}_{C}$.

By Lemma 2.5(a), the composition $\operatorname{Hom}_{R}\left(C, v_{M}\right) \circ \mu_{\mathrm{Hom}_{R}(C, M)}$ is the identity map on $\operatorname{Hom}_{R}(C, M)$. Since $\operatorname{Hom}_{R}(C, M)$ is in $\mathscr{A}_{C}$, the map $\mu_{\mathrm{Hom}_{R}(C, M)}$ is an isomorphism, and so $\operatorname{Hom}_{R}\left(C, v_{M}\right)$ is also an isomorphism. Setting $K=\operatorname{ker}\left(v_{M}\right)$, it follows that $\operatorname{Hom}_{R}(C, K)=0$, and so by [7, (3.6)], $K=0$. Thus, the map $v_{M}$ is injective, hence an isomorphism by Corollary 2.7(a).

Recall that the class $\mathscr{B}_{C}$ contains all modules of finite injective dimension and the class $\mathscr{A}_{C}$ contains all modules of finite projective dimension; see 1.9. By virtue of Theorem 2.8, we now obtain additional examples of modules in $\mathscr{A}_{C}$ and $\mathscr{B}_{C}$.

Corollary 2.9. Let $C$ be a semidualizing $R$-module and $M$ an $R$-module.

(a) If $\mathscr{P}_{C}-\operatorname{pd}_{R}(M)$ is finite, then $M$ is in $\mathscr{B}_{C}$.

(b) If $\mathscr{I}_{C}-\operatorname{id}_{R}(M)$ is finite, then $M$ is in $\mathscr{A}_{C}$.

Proof. We prove only part (a). Assume $\mathscr{P}_{C}-\operatorname{pd}_{R}(M)$ is finite, and let $X^{+}$ be an augmented proper $\mathscr{P}_{C}$-resolution of $M$. By Lemma 2.1(a), the complex $\operatorname{Hom}_{R}\left(C, X^{+}\right)$is a bounded projective resolution of $\operatorname{Hom}_{R}(C, M)$. By 1.9(b) $\operatorname{Hom}_{R}(C, M)$ is in $\mathscr{A}_{C}$ so Theorem 2.8(a) implies that $M$ is in $\mathscr{B}_{C}$.

This yields the following key result.

Corollary 2.10. Let $C$ be a semidualizing $R$-module and $M$ an $R$-module.

(a) The inequality $\mathscr{P}_{C^{-}} \operatorname{pd}_{R}(M) \leqslant n$ holds if and only if there is an exact sequence

$$
0 \rightarrow C \otimes_{R} P_{n} \rightarrow \cdots \rightarrow C \otimes_{R} P_{0} \rightarrow M \rightarrow 0
$$

with each $P_{i}$ a projective $R$-module. 
(b) The inequality $\mathscr{I}_{C}-\mathrm{id}_{R}(M) \leqslant n$ holds if and only if there is an exact sequence

$$
0 \rightarrow M \rightarrow \operatorname{Hom}_{R}\left(C, I^{0}\right) \rightarrow \cdots \rightarrow \operatorname{Hom}_{R}\left(C, I^{n}\right) \rightarrow 0
$$

with each $I^{i}$ an injective $R$-module.

Proof. The "only if" direction of each statement follows immediately from Corollaries 2.4 and 2.9. For the other implications, use dimension shifting 1.7 to see that any exact sequence of the given form is an augmented proper $\mathscr{P}_{C^{-}}$ projective or $\mathscr{I}_{C}$-injective resolution of $M$.

We now investigate how $\mathscr{P}_{C}$-projective and projective dimension relate.

TheOREM 2.11. Let $C$ be a semidualizing $R$-module. The following equalities hold.

(a) $\operatorname{pd}_{R}(M)=\mathscr{P}_{C}-\operatorname{pd}_{R}\left(C \otimes_{R} M\right)$

(b) $\mathscr{I}_{C}-\mathrm{id}_{R}(M)=\mathrm{id}_{R}\left(C \otimes_{R} M\right)$

(c) $\mathscr{P}_{C^{-}}-\operatorname{pd}_{R}(M)=\operatorname{pd}_{R}\left(\operatorname{Hom}_{R}(C, M)\right)$

(d) $\operatorname{id}_{R}(M)=\mathscr{I}_{C}-\operatorname{id}_{R}\left(\operatorname{Hom}_{R}(C, M)\right)$

Proof. We prove only part (a). Assume $\operatorname{pd}_{R}(M)=s<\infty$ and consider an augmented projective resolution of $\mathrm{M}$

$$
X=0 \rightarrow P_{s} \rightarrow P_{s-1} \rightarrow \cdots \rightarrow P_{0} \rightarrow M \rightarrow 0 .
$$

By 1.9(b), one has $M \in \mathscr{A}_{C}$ and so $\operatorname{Tor}_{\geqslant 1}^{R}(C, M)=0$. Thus, the complex

$$
C \otimes_{R} X=0 \rightarrow C \otimes_{R} P_{s} \rightarrow C \otimes_{R} P_{t-1} \rightarrow \cdots \rightarrow C \otimes_{R} P_{0} \rightarrow C \otimes_{R} M \rightarrow 0
$$

is exact and thus an augmented proper $\mathscr{P}_{C}$-projective resolution of $C \otimes_{R} M$. Note that properness can be shown by using 1.7 , or as a special case of $[13$, (4.4)]. This provides an inequality $s \geqslant \mathscr{P}_{C}-\mathrm{pd}_{R}\left(C \otimes_{R} M\right)$. Conversely, assume that $\mathscr{P}_{C}-\operatorname{pd}_{R}\left(C \otimes_{R} M\right)=t<\infty$. By Corollary 2.10(a), there is an augmented exact proper $\mathscr{P}_{C}$-resolution of $C \otimes_{R} M$

$$
X^{+}=0 \rightarrow C \otimes_{R} P_{t} \rightarrow C \otimes_{R} P_{t-1} \rightarrow \cdots \rightarrow C \otimes_{R} P_{0} \rightarrow C \otimes_{R} M \rightarrow 0 .
$$

Thus, the complex $\operatorname{Hom}_{R}\left(C, X^{+}\right)$is exact. Corollary 2.9 implies that $C \otimes_{R}$ $M$ is in $\mathscr{B}_{C}$ and Theorem 2.8 then implies that $M$ is in $\mathscr{A}_{C}$. Thus, $\mu_{M}$ is an isomorphism. Since each $\mu_{P_{i}}$ is also an isomorphism, $\operatorname{Hom}_{R}\left(C, X^{+}\right)$is isomorphic to an exact sequence of the form

$$
0 \rightarrow P_{t} \rightarrow P_{t-1} \rightarrow \cdots \rightarrow P_{0} \rightarrow M \rightarrow 0
$$


Thus, $\operatorname{pd}_{R}(M) \leqslant t=\mathscr{P}_{C^{-}} \operatorname{pd}_{R}\left(C \otimes_{R} M\right)$.

Note that by assembling the information above, we get the following extension of the Foxby equivalence [1].

THEOREM 2.12 (Foxby equivalence). Let $C$ be a semidualizing $R$-module, and let $n$ be a non-negative integer. Set $\widehat{\mathscr{P}}_{C}(R)_{\leqslant n}, \widehat{\mathscr{P}}(R)_{\leqslant n}, \widehat{\mathscr{I}}_{C}(R)_{\leqslant n}$, and $\widehat{\mathscr{I}}(R)_{\leqslant n}$ to be the classes of modules of $C$-projective, projective, $C$-injective and injective dimension of at most $n$, respectively. Then there are equivalences of categories

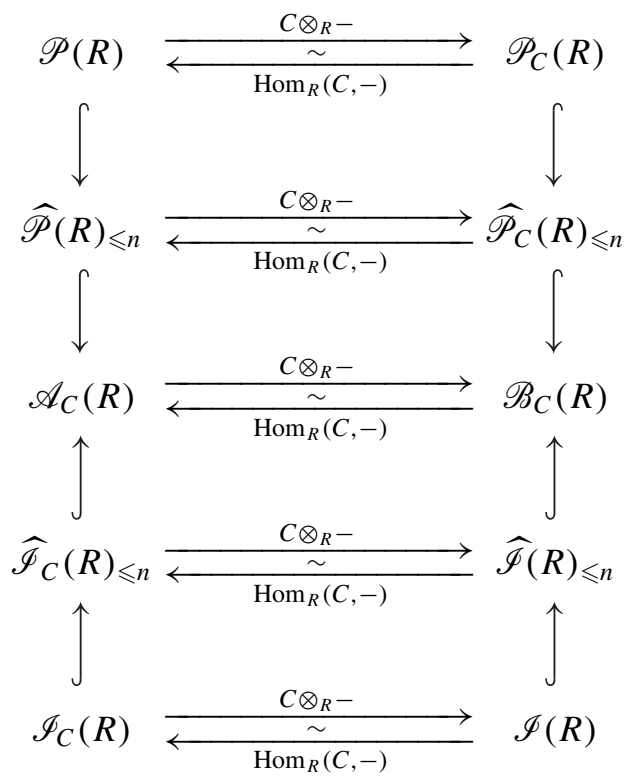

\section{Vanishing of relative cohomology and consequences}

In this section, we investigate how vanishing of the relative cohomology functors $\operatorname{Ext}_{\mathscr{P}_{C}}^{i}(M,-)$ and $\operatorname{Ext}_{\mathscr{I}_{C}}^{i}(-, N)$, respectively, characterizes the finiteness of $\mathscr{P}_{C^{-}} \mathrm{pd}_{R}(M)$ and $\mathscr{I}_{C^{-}}$id ${ }_{R}(N)$. Our proofs use specific properties of semidualizing modules and so do not carry over directly to other relative settings.

TheOREM 3.1. Let $C$ be a semidualizing $R$-module and $M$ an $R$-module.

(a) The following are equivalent.

(i) $\operatorname{Ext}_{\mathscr{P}_{C}}^{1}(M,-)=0$

(ii) $\operatorname{Ext}_{\mathscr{P}_{C}}^{\geqslant 1}(M,-)=0$

(iii) $M$ is $C$-projective

(a) The following are equivalent.

(i) $\operatorname{Ext}_{\mathscr{I}_{C}}^{1}(-, M)=0$ 
(ii) $\operatorname{Ext}_{\mathscr{I}_{C}}^{\geqslant 1}(-, M)=0$

(iii) $M$ is $C$-injective

Proof. We prove part (a); part (b) is dual.

(iii) $\Rightarrow$ (ii): If $M$ is $C$-projective, then the complex

$$
\cdots \rightarrow 0 \rightarrow M \stackrel{=}{\longrightarrow} M \rightarrow 0
$$

is an augmented proper $\mathscr{P}_{C}$-resolution of $M$ and so $\operatorname{Ext}_{\mathscr{P}_{C}}^{\geqslant 1}(M,-)=0$.

(ii) $\Rightarrow$ i) is immediate.

(i) $\Rightarrow$ (iii): Let

$$
X=\cdots \stackrel{d_{2}}{\longrightarrow} C \otimes_{R} P_{1} \stackrel{d_{1}}{\longrightarrow} C \otimes_{R} P_{0} \stackrel{d_{0}}{\longrightarrow} M \rightarrow 0
$$

be an augmented proper $\mathscr{P}_{C}$-resolution of $M$. Let $K_{0}$ be the kernel of $d_{0}$, and let $\beta: K_{0} \rightarrow C \otimes_{R} P_{0}$ be the inclusion map. There is a homomorphism $\alpha: C \otimes_{R} P_{1} \rightarrow K_{0}$ such that $d_{1}=\beta \alpha$. Noting that $\beta \alpha d_{2}=d_{1} d_{2}=0$, the injectivity of $\beta$ implies that $\alpha d_{2}=0$. Since $\operatorname{Ext}_{\mathscr{P}_{C}}^{1}\left(M, K_{0}\right)=0$, the induced sequence

$$
\operatorname{Hom}_{R}\left(C \otimes_{R} P_{0}, K_{0}\right) \rightarrow \operatorname{Hom}_{R}\left(C \otimes_{R} P_{1}, K_{0}\right) \rightarrow \operatorname{Hom}_{R}\left(C \otimes_{R} P_{2}, K_{0}\right)
$$

is exact. Hence, there exists $\xi \in \operatorname{Hom}_{R}\left(C \otimes_{R} P_{0}, K_{0}\right)$ such that $\alpha=\xi d_{1}=$ $\xi \beta \alpha$. There is an equality $\operatorname{Hom}_{R}(C, \alpha)=\operatorname{Hom}_{R}(C, \xi) \circ \operatorname{Hom}_{R}(C, \beta) \circ$ $\operatorname{Hom}_{R}(C, \alpha)$ and $\operatorname{so}_{H^{\prime}}(C, \xi) \circ \operatorname{Hom}_{R}(C, \beta)=\operatorname{id}_{H^{\prime}}\left(C, K_{0}\right)$, as $\operatorname{Hom}_{R}(C$, $\alpha$ ) is surjective. Therefore, the exact sequence (which is exact by properness of $X$ )

$$
0 \rightarrow \operatorname{Hom}_{R}\left(C, K_{0}\right) \stackrel{\operatorname{Hom}_{R}(C, \beta)}{\longrightarrow} \operatorname{Hom}_{R}\left(C, C \otimes_{R} P_{0}\right) \rightarrow \operatorname{Hom}_{R}(C, M) \rightarrow 0
$$

splits. Since $\operatorname{Hom}_{R}\left(C, C \otimes_{R} P_{0}\right) \cong P_{0}$ is $R$-projective, so is $\operatorname{Hom}_{R}(C, M)$. Theorem 2.11 and Corollary 2.10(a) now imply that $M$ is $C$-projective.

Using dimension shifting, we have the following extension of the previous result.

Theorem 3.2. Let $C$ be a semidualizing $R$-module, let $n$ be a non-negative integer, and let $M, N$ be $R$-modules.

(a) The following are equivalent.
(i) $\operatorname{Ext}_{\mathscr{P}_{C}}^{n+1}(M,-)=0$
(ii) $\operatorname{Ext}_{\mathscr{P}_{C}}^{\geqslant n+1}(M,-)=0$
(iii) $\mathscr{P}_{C^{-}} \operatorname{pd}_{R}(M) \leqslant n$. 
(b) The following are equivalent.

(i) $\operatorname{Ext}_{\mathscr{I}_{C}}^{n+1}(-, N)=0$

(ii) $\operatorname{Ext}_{\mathscr{I}_{C}}^{\geqslant n+1}(-, N)=0$

(iii) $\mathscr{I}_{C}-\operatorname{id}_{R}(N) \leqslant n$.

The preceding two results imply the following.

Corollary 3.3. Let $C$ be a semidualizing $R$-module, $M$ an $R$-module and $n \geqslant 0$.

(a) The following are equivalent.

(i) There exists an augmented proper $\mathscr{P}_{C}$-projective resolution $X^{+}$of $M$ such that $\Omega_{n}^{X^{+}}$is $C$-projective.

(ii) Every augmented proper $\mathscr{P}_{C}$-projective resolution $X^{+}$of $M$ has the property that $\Omega_{n}^{X^{+}}$is $C$-projective.

(b) The following are equivalent.

(i) There exists an augmented proper $\mathscr{I}_{C}$-injective resolution $Y^{+}$of $M$ such that $\Omega_{Y^{+}}^{n}$ is $C$-injective.

(ii) Every augmented proper $\mathscr{I}_{C}$-injective resolution $Y^{+}$of $M$ has the property that $\Omega_{Y^{+}}^{n}$ is $C$-injective.

We conclude this section by showing that if any two of three modules in a short exact sequence have finite $\mathscr{P}_{C}$-projective dimension then so does the third. Note that the standard constructions (using Horseshoe Lemmas, mapping cones, etc.) that show this result when $C=R$ can be used in this setting. However, we offer a shorter proof that uses the classical result.

Proposition 3.4. Let $C$ be a semidualizing $R$-module. Consider an exact sequence of $R$-modules

$$
0 \rightarrow M^{\prime} \rightarrow M \rightarrow M^{\prime \prime} \rightarrow 0
$$

If any two of the modules have finite $\mathscr{P}_{C}$-projective dimension, respectively $\mathscr{I}_{C}$-injective dimension, then so does the third.

Proof. Assume that two of the modules $M, M^{\prime}, M^{\prime \prime}$ have finite $\mathscr{P}_{C}$-projective dimension. By Corollary 2.9, these two modules are in $\mathscr{B}_{C}$. By 1.9(a), this forces all of the modules $M, M^{\prime}, M^{\prime \prime}$ to be in $\mathscr{B}_{C}$. Thus, the complex

$$
0 \rightarrow \operatorname{Hom}_{R}\left(C, M^{\prime}\right) \rightarrow \operatorname{Hom}_{R}(C, M) \rightarrow \operatorname{Hom}_{R}\left(C, M^{\prime \prime}\right) \rightarrow 0
$$

is exact. By Theorem 2.11(c), two of the above Hom modules have finite projective dimension and hence so does the third. Theorem 2.11(c) implies that all of $M, M^{\prime}, M^{\prime \prime}$ have finite $P_{C}$-projective dimension. The other assertion is dual. 


\section{Comparing relative and absolute cohomology}

In this section we investigate the interplay between absolute Ext and the relative cohomologies $\operatorname{Ext}_{\mathscr{P}_{C}}$ and $\operatorname{Ext}_{\mathscr{I}_{C}}$. Under certain circumstances, they agree with their corresponding absolute counterparts.

Theorem 4.1. Let $C$ be a semidualizing $R$-module, and let $M$ and $N$ be $R$-modules. There exist isomorphisms.

$$
\begin{aligned}
& \operatorname{Ext}_{\mathscr{P}_{C}}^{i}(M, N) \cong \operatorname{Ext}_{R}^{i}\left(\operatorname{Hom}_{R}(C, M), \operatorname{Hom}_{R}(C, N)\right) \\
& \operatorname{Ext}_{\mathscr{I}_{C}}^{i}(M, N) \cong \operatorname{Ext}_{R}^{i}\left(C \otimes_{R} M, C \otimes_{R} N\right)
\end{aligned}
$$

Proof. We prove only part (a). Let $X^{+}$be an augmented proper $\mathscr{P}_{C^{-}}$ resolution of $M$. By Lemma 2.1, the complex $\operatorname{Hom}_{R}\left(C, X^{+}\right)$is an augmented projective resolution of $\operatorname{Hom}_{R}(C, M)$. Thus, the equalities below hold by definition

$$
\begin{aligned}
\operatorname{Ext}_{R}^{i} & \left(\operatorname{Hom}_{R}(C, M), \operatorname{Hom}_{R}(C, N)\right) \\
& =\mathrm{H}^{i}\left(\operatorname{Hom}_{R}\left(\operatorname{Hom}_{R}(C, X), \operatorname{Hom}_{R}(C, N)\right)\right) \\
& \cong \mathrm{H}^{i}\left(\operatorname{Hom}_{R}\left(C \otimes_{R} \operatorname{Hom}_{R}(C, X), N\right)\right) \\
& \cong \mathrm{H}^{i}\left(\operatorname{Hom}_{R}(X, N)\right) \\
& =\operatorname{Ext}_{\mathscr{P}_{C}}^{i}(M, N),
\end{aligned}
$$

while the isomorphisms follow from adjunction and the containment $\mathscr{P}_{C} \subseteq$ $\mathscr{B}_{C}$.

With appropriate Auslander and Bass class assumptions, the aforementioned relative cohomology modules agree precisely with the absolute Ext.

Corollary 4.2. Let $C$ be a semidualizing $R$-module, and let $M, N$ be $R$-modules.

(a) If $M$ and $N$ are in $\mathscr{B}_{C}$, then $\operatorname{Ext}_{\mathscr{P}_{C}}^{i}(M, N) \cong \operatorname{Ext}_{R}^{i}(M, N)$ for all $i$.

(b) If $M$ and $N$ are in $\mathscr{A}_{C}$, then $\operatorname{Ext}_{\mathscr{I}_{C}}^{i}(M, N) \cong \operatorname{Ext}_{R}^{i}(M, N)$ for all $i$.

Proof. We prove only part(a). Let Tot $X$ denote the total complex of a double complex $X$. Let $P^{+}$be an augmented projective resolution of $\operatorname{Hom}_{R}(C$, $M$ ), and let $I^{+}$be an augmented injective resolution of $N$. Since $M$ and $N$ are in $\mathscr{B}_{C}$, the complexes $C \otimes_{R} P^{+}$and $\operatorname{Hom}_{R}\left(C, I^{+}\right)$are exact. There is an isomorphism

$$
\operatorname{Hom}_{R}\left(C \otimes_{R} P, I\right) \cong \operatorname{Hom}_{R}\left(P, \operatorname{Hom}_{R}(C, I)\right)
$$


of double complexes. This provides the second isomorphism below

$$
\begin{aligned}
\operatorname{Ext}_{R}^{i}(M, N) & \cong \mathrm{H}^{i}\left(\operatorname{Tot}_{\operatorname{Hom}_{R}}\left(C \otimes_{R} P, I\right)\right) \\
& \cong \mathrm{H}^{i}\left(\operatorname{Tot}_{\operatorname{Hom}_{R}}\left(P, \operatorname{Hom}_{R}(C, I)\right)\right) \\
& \cong \operatorname{Ext}_{R}^{i}\left(\operatorname{Hom}_{R}(C, M), \operatorname{Hom}_{R}(C, N)\right) \\
& \cong \operatorname{Ext}_{\mathscr{P}_{C}}^{i}(M, N),
\end{aligned}
$$

while the last isomorphism follows from Theorem 4.1.

\section{Further parallels between the classical and relative theories}

The results of the previous sections demonstrate that there is a tight connection between modules of finite $\mathscr{P}_{C}$-projective dimension and modules of finite projective dimension. In this section we indicate how the machinery developed above allows us to extend many classical results to this new setting. We begin by showing that $\mathscr{P}_{C}$-projective dimension has the ability to detect when a ring is regular.

Proposition 5.1. Let $(R, \mathfrak{m}, k)$ be a noetherian, local ring and $C$ a semidualizing $R$-module. The following are equivalent.

(i) $\mathscr{P}_{C}-\operatorname{pd}_{R}(M)$ is finite for all $R$-modules $M$.

(ii) $\mathscr{P}_{C}-\operatorname{pd}_{R}(k)$ is finite.

(iii) $R$ is regular.

Proof. (i) $\Longrightarrow$ (ii) is trivial.

(ii) $\Longrightarrow$ (iii) Since $\mathscr{P}_{C}-\mathrm{pd}_{R}(k)$ is finite, Lemma 2.1 implies $\operatorname{pd}_{R}\left(\operatorname{Hom}_{R}(C\right.$, $k)$ ) is finite. Since $\operatorname{Hom}_{R}(C, k)$ is a nonzero $k$-vector space, $\operatorname{pd}_{R}(k)$ is finite. Thus, $R$ is regular.

(iii) $\Longrightarrow$ (i) Since $R$ is regular, the only semidualizing $R$-module is $R$ itself. Thus, $C=R$ so this follows from the Auslander-Buchsbaum-Serre theorem.

Our methods also apply to bounded complexes of $C$-projective modules, as the next result shows.

Proposition 5.2 (New Intersection Theorem for complexes of $C$-projective modules). Let $(R, \mathfrak{m})$ be a noetherian local ring and $C$ a semidualizing $R$ module. If there exists a non-exact complex

$$
X=0 \rightarrow C^{\alpha_{s}} \rightarrow C^{\alpha_{s-1}} \rightarrow \cdots \rightarrow C^{\alpha_{1}} \rightarrow C^{\alpha_{0}} \rightarrow 0
$$

with $\ell_{R}\left(\mathrm{H}_{i}(X)\right)$ finite for all $i$, then $s \geqslant \operatorname{dim}(R)$. 
Proof. First, note that the complex

$$
\operatorname{Hom}_{R}(C, X)=0 \rightarrow R^{\alpha_{s}} \rightarrow R^{\alpha_{s-1}} \rightarrow \cdots \rightarrow R^{\alpha_{1}} \rightarrow R^{\alpha_{0}} \rightarrow 0
$$

is non-exact. Indeed, if it were exact, then it would split, forcing the complex $C \otimes_{R} \operatorname{Hom}_{R}(C, X) \cong X$ to be exact, a contradiction.

Now fix a prime $\mathfrak{p} \neq \mathfrak{m}$. Since the homology of the complex $X$ has support equal to $\mathfrak{m}$, the complex $X_{\mathfrak{p}}$ is exact. This forces the complex $\operatorname{Hom}_{R_{\mathfrak{p}}}\left(C_{\mathfrak{p}}, X_{\mathfrak{p}}\right)$ to be exact, as $\operatorname{Ext}_{R}^{\geqslant 1}(C, C)=0$. This forces $\mathrm{H}_{i}\left(\operatorname{Hom}_{R}(C, X)\right)_{\mathfrak{p}} \cong \mathrm{H}_{i}\left(\operatorname{Hom}_{R_{\mathfrak{p}}}\left(C_{\mathfrak{p}}\right.\right.$, $\left.\left.X_{\mathfrak{p}}\right)\right)=0$ for all $i$. Thus, $\ell_{R}\left(H_{i}\left(\operatorname{Hom}_{R}(C, X)\right)\right)<\infty$ for all $i$. The New Intersection Theorem now implies that $s \geqslant \operatorname{dim}(R)$.

Next, we extend Bass' result that a ring is noetherian if and only if the class of injective $R$-modules is closed under direct sums.

Proposition 5.3. Let $R$ be a commutative ring and $C$ a semidualizing $R$ module. The ring $R$ is noetherian if and only if the class $\mathscr{I}_{C}$ is closed under direct sums.

Proof. Assume $R$ is noetherian. Let $\left\{I_{\lambda}\right\}$ be a collection of injective $R$ modules. Since $C$ is finitely presented, there is an isomorphism

$$
\coprod_{\lambda} \operatorname{Hom}_{R}\left(C, I_{\lambda}\right) \cong \operatorname{Hom}_{R}\left(C, \coprod_{\lambda} I_{\lambda}\right)
$$

and the desired result follows by Bass' result.

Conversely, assume the class $\mathscr{I}_{C}$ is closed under direct sums. Let $\left\{I_{\lambda}\right\}$ be a collection of injective modules so that $\left\{\operatorname{Hom}_{R}\left(C, I_{\lambda}\right)\right\}$ is a collection of $C$ injective modules. By assumption, there is an isomorphism

$$
\coprod \operatorname{Hom}_{R}\left(C, I_{\lambda}\right) \cong \operatorname{Hom}_{R}(C, J)
$$

for some injective $J$. This provides the third isomorphism below. The first and fourth isomorphisms follow from the fact that any injective module is in $\mathscr{B}_{C}$. The second is by the commutativity of tensor products and coproducts.

$$
\begin{aligned}
\coprod I_{\lambda} & \cong \coprod C \otimes_{R} \operatorname{Hom}_{R}\left(C, I_{\lambda}\right) \\
& \cong C \otimes_{R} \coprod\left(\operatorname{Hom}_{R}\left(C, I_{\lambda}\right)\right) \\
& \cong C \otimes_{R} \operatorname{Hom}_{R}(C, J) \\
& \cong J
\end{aligned}
$$

In particular, $\coprod_{\lambda}$ is injective. 
Finally, we provide an example in which this technique does not seem to provide a straightforward way in which to extend a classical result. Consider the following:

Question 5.4. Let $(R, \mathrm{~m}, k)$ be a local, Cohen-Macaulay ring admitting a dualizing module $D$. Let $C$ be a semidualizing $R$-module. If there exists an $R$-module $M$ of finite depth with finite $\mathscr{P}_{C}$-projective dimension and finite $\mathscr{I}_{C}$-injective dimension, must $R$ be Gorenstein?

Note that, as we try to apply the aforementioned techniques, we see that $\operatorname{Hom}_{R}(C, M)$ has finite projective dimension, while $C \otimes_{R} M$ has finite injective dimension. To apply the classical result, we need a single module that has both finite projective dimension and finite injective dimension.

ACKNOWLEDGMents. The authors would like to thank Lars Winther Christensen, Sean Sather-Wagstaff and the referee for helpful comments and suggestions.

\section{REFERENCES}

1. Avramov, L. L., and Foxby, H.-B., Ring homomorphisms and finite Gorenstein dimension, Proc. London Math. Soc. (3) 75 (1997), 241-270.

2. Enochs, E., and Holm, H., Cotorsion pairs associated with Auslander categories, Israel J. Math., to appear, available from arXiv:math.AC/0609291.

3. Enochs, E. E., and Jenda, O. M. G., Relative Homological Algebra, de Gruyter Expositions in Math. 30, de Gruyter, Berlin 2000.

4. Foxby, H.-B., Gorenstein modules and related modules, Math. Scand. 31 (1973), 267-284.

5. Golod, E. S., G-dimension and generalized perfect ideals, Algebraic Geometry and its Applications, Trudy Mat. Inst. Steklov 165 (1984), 62-66.

6. Hartshorne, R., Local Cohomology, Lecture Notes in Math. 41, Springer, Berlin 1967.

7. Holm, H., and White, D., Foxby equivalence over associative rings, J. Math. Kyoto Univ. 47 (2007), 781-808.

8. Holm, H., Gorenstein homological dimensions, J. Pure Appl. Algebra 189 (2004), 167-193.

9. Sather-Wagstaff, S., Sharif, T., and White, D., Comparison of relative cohomology theories with respect to semidualizing modules, Math. Z. 264 (2010), 571-600.

10. Sather-Wagstaff, S., Sharif, T., and White, D., Gorenstein cohomology in abelian categories, J. Math. Kyoto Univ. 48 (2008), 571-596.

11. Sather-Wagstaff, S., Sharif, T., and White, D., Stability of Gorenstein categories, J. London Math. Soc. (2) 77 (2008), 481-502.

12. Vasconcelos, W. V., Divisor theory in module categories, North-Holland Math. Studies 14, Notas de Matemática 53, North-Holland, Amsterdam, 1974. 
13. White, D., Gorenstein projective dimension with respect to a semidualizing module, J. Commut. Algebra, to appear, available from arXiv:math.AC/0611711.

\author{
DEPARTMENT OF MATHEMATICAL SCIENCES \\ FACULTY OF SCIENCE \\ SHINSHU UNIVERSITY \\ 3-1-1 ASAHI, MATSUMOTO \\ NAGANO 390-8621 \\ JAPAN \\ E-mail: takahasi@math.shinshu-u.ac.jp
}

\author{
DEPARTMENT OF MATHEMATICS \\ UNIVERSITY OF COLORADO DENVER \\ CAMPUS BOX 170 \\ P.O. BOX 173364 \\ DENVER, CO 80217-3364 \\ USA \\ E-mail: diana.white@ucdenver.edu
}

